\title{
Distributed Aperture Imaging with Multiple Transmitters in Complex Environments
}

\author{
T. Varslot ${ }^{a)}$, B. $\operatorname{Yazicl}^{a)}$, M. Cheney ${ }^{b)}$ \\ a) Dept. Electrical, Computer and Systems Engineering, Rensselaer Polytechnic Institute, \\ Troy, NY, USA \\ b) Dept. Mathematical Sciences, Rensselaer Polytechnic Institute, Troy, NY, USA
}

\begin{abstract}
We present a new image reconstruction method for distributed apertures operating in complex environments with additive non-stationary noise. Our method is capable of exploiting information that we might have about: multipath scattering in the environment; statistics of the objects to be imaged; statistics of the additive nonstationary noise. The aperture elements are distributed spatially in an arbitrary fashion, and can be several hundred wavelengths apart. Furthermore, our method facilitates multiple transmit apertures which operate simultaneously, and is thus capable of handling a true multi-transmit-multi-receive scenario. We derive a set of basis functions which is adapted to the given operating environment and sensor distribution. By selecting an appropriate subset of these basis functions we obtain a sub-space reconstruction which is optimal in the sense of obtaining the minimum-mean-square-error for the reconstructed image. Furthermore, as this subspace determines which details will be visible in the reconstructed image, it provides a tool for evaluating the sensor locations against the objects that we would like to see in the image. The implementation of our reconstruction takes the form of a filter bank which is applied to the pulse-echo measurements. This processing can be performed independently on the measurements obtained from each receiving element. Our approach is therefore well suited for parallel implementation, and can be performed in a distributed manner in order to reduce the required communication bandwidth between each receiver and the location where the results are merged into the final image. We present numerical simulations which illustrate capabilities of our method.
\end{abstract}

Keywords: distributed aperture, multi-path scattering, sparse aperture, image reconstruction, pulse-echo imaging

\section{INTRODUCTION}

Pulse-echo imaging covers a wide range of applications from ultrasound and micro-wave imaging, to sonar and radar imaging, and is performed by transmitting a waveform, and recording the resulting scattering from the object to be imaged. From these scattering measurements, we form an image of the object. The image is a spatially resolved map of the object's scattering strength. ${ }^{1}$

We focus our attention to extremely sparse arrays where the array elements can be located several hundred wavelengths apart. Such an array is referred to as a distributed aperture. ${ }^{2}$ An important issue for such apertures is that the region of interest no longer is in the far-field of the array. This introduces range dependence in the scattering measurements which cannot be ignored. ${ }^{3,4}$ By using a physics-based measurement model we directly account for this range-dependence.

In our work we use wide-band transmit waveforms, and exploit multi-path scattering in order to image extended objects using a distributed aperture. We derive an inversion technique which is optimal in the sense that it yields the minimum-norm solution which attains the minimum $\mathrm{L}^{2}$-error in the reconstructed image. In doing so we allow for multiple transmitters and receivers to be activated simultaneously. ${ }^{5-8}$ Furthermore,

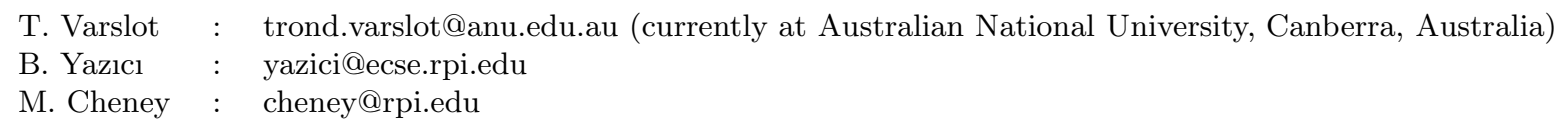

Algorithms for Synthetic Aperture Radar Imagery XV, edited by Edmund G. Zelnio, Frederick D. Garber Proc. of SPIE Vol. 6970, 69700J, (2008) · 0277-786X/08/\$18 · doi: 10.1117/12.777504 


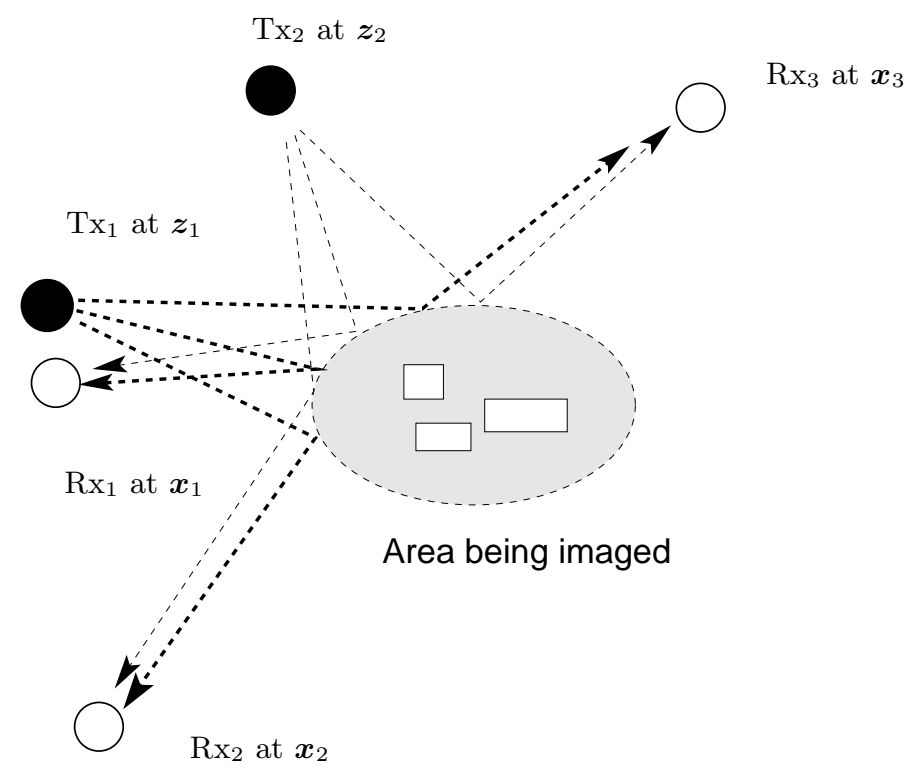

Figure 1. Distributed aperture with two transmitting elements (black circles) and three receiving elements (white circles). The transmitting elements $\mathrm{Tx}_{1}$ and $\mathrm{Tx}_{2}$ are located at spatial positions $\boldsymbol{z}_{1}$ and $\boldsymbol{z}_{2}$, respectively. The receiving elements $\mathrm{Rx}_{1}, \mathrm{Rx}_{2}$ and $\mathrm{Rx}_{3}$ are located at positions $\boldsymbol{x}_{1}, \boldsymbol{x}_{2}$ and $\boldsymbol{x}_{3}$. Arrows indicate that scattering as a result of a waveform transmitted from element $\mathrm{Tx}_{1}$ will be measured on all receive elements; similarly for $\mathrm{Tx}_{2}$.

we do not make additional assumptions about being able to separate the waveforms from each transmitter by orthogonality..$^{5,9}$

In Devaney et al. ${ }^{10,11}$ a similar reconstruction method was presented under the assumption that the impulse response from all pairs of transmitters and receivers could be obtained. We do not make this assumption, and account for transmit waveform diversity by conducting our analysis in the continuous-time domain. Our approach enables us to state the reconstruction algorithm explicitly in terms of processing of the time-domain scattering measurements. Also in Yazıcı et al. ${ }^{12}$ the general approach to reconstruction is similar. However, by employing the affine Fourier transform to perform the actual inversion, that work inherently relies on the freespace propagation model, and deals with one transmitter and one receiver which are assumed to be co-located. There are no such assumptions in our current work. In fact, the ability to exploit multi-path propagation is one of the features of our work; our work generalizes the inversion technique presented in ${ }^{12}$ to distributed apertures in a multi-path environment.

\section{FORWARD MODEL}

\subsection{Distributed Apertures}

We consider an array consisting of $m$ transmitting elements and $n$ receiving elements. The array elements are arbitrarily distributed, and can be several hundred wavelengths apart. These spatially distributed array elements constitute our aperture. In our development we allow for an arbitrary spatial distribution of the elements. To reflect this spatial flexibility, we refer to the array as a distributed aperture. ${ }^{2}$ An illustration of the distributed nature of an array with two transmitting elements and three receiving elements is shown in Fig. 1.

In order to exploit the spatial diversity we want to allow for transmitting different waveforms from each array element. Let $s_{j}(t)$ denote the waveform which emanates from the $j^{\text {th }}$ element. The transmit waveforms are arranged in a transmit vector $\boldsymbol{s}(t)$

$$
\boldsymbol{s}(t):=\left[s_{1}(t), \ldots, s_{m}(t)\right]^{\mathrm{T}} .
$$

Note that we use a bold-face italic font for vector quantities. 
Similarly, if the measured scattering at the $i^{\text {th }}$ receive element is denoted $m_{i}(t)$, then the signal which is collected by the array may be arranged in a measurement vector $\boldsymbol{m}(t)$

$$
\boldsymbol{m}(t):=\left[m_{1}(t), \ldots, m_{n}(t)\right]^{\mathrm{T}} .
$$

\subsection{Channel Model}

Any object with properties which deviate from a constant (homogeneous) background will produce scattering. However, scattering from an object for which location and scattering strength is known does not contribute new information. We therefore use the known objects to define a background medium. Scattering is defined in terms of deviations from this background. Our ability to observe the additional objects depends on how much the relevant physical properties deviate from the background. We denote this deviation by the reflectivity function $V(\boldsymbol{x})$.

For our current work we will assume that the propagating wave field $u$ is described by the following scalar wave equation

$$
\nabla^{2} u-\frac{1}{c^{2}} \partial_{t}^{2} u=V \partial_{t}^{2} u .
$$

Here $c$ is the propagation speed in the background medium. This model gives a good description for acoustic pressure waves of moderate amplitude, as well as electromagnetic wave propagation through a medium such dry air, where polarization effects may be neglected.

Our model for the scattering is given in terms of the Green's function $g(\boldsymbol{x}, \boldsymbol{y}, t)$ for the background medium. The Green's function is the response measured at position $\boldsymbol{x}$ due to an impulse $\delta(t)$ transmitted from position $\boldsymbol{y}$, i.e., a solution of

$$
\nabla_{\boldsymbol{x}}^{2} g-\frac{1}{c^{2}} \partial_{t}^{2} g=\delta(t) \delta(|\boldsymbol{x}-\boldsymbol{y}|)
$$

We will now let the $j^{\text {th }}$ transmit element be located at position $\boldsymbol{z}^{j}$, and the $i^{\text {th }}$ receive element be located at position $\boldsymbol{x}^{i}$ (see Fig. 1). Furthermore, we define the matrix $G(\boldsymbol{y}, t)$ with matrix elements

$$
G_{i j}(\boldsymbol{y}, t):=\int g\left(\boldsymbol{z}_{j}, \boldsymbol{y}, \tau^{\prime}\right) \partial_{t}^{2} g\left(\boldsymbol{y}, \boldsymbol{x}_{i}, t-\tau^{\prime}\right) \mathrm{d} \tau^{\prime} .
$$

If we neglect multiple reflections involving the object to be imaged, we may express the measurement vector defined in Eq. (2) as

$$
\boldsymbol{m}(t)=\int G(\boldsymbol{y}, t-\tau) V(\boldsymbol{y}) \mathrm{d} \boldsymbol{y} \boldsymbol{s}(\tau) \mathrm{d} \tau .
$$

Integration in Eq. (6) is understood to be element wise

$$
m_{i}(t)=\sum_{j=1}^{m} \int G_{i j}(\boldsymbol{y}, t-\tau) V(\boldsymbol{y}) \mathrm{d} \boldsymbol{y} s_{j}(\tau) \mathrm{d} \tau .
$$

We refer to the mapping from transmit vector $\boldsymbol{s}(t)$ to measurement vector $\boldsymbol{m}(t)$ as the channel, and denote it by $\mathcal{H}(V)$.

$$
\boldsymbol{m}(t):=\mathcal{H}(V) \boldsymbol{s}(t)
$$




\subsection{Problem Statement}

The channel $\mathcal{H}(V)$ is a linear mapping from transmit vectors to measurement vectors. We now define $\mathcal{H}$ as the linear mapping which takes the reflectivity function $V$ to the channel $\mathcal{H}(V)$. The kernel of $\mathcal{H}$ is in this case $G$ from Eq. (5). Furthermore, if we identify $\mathcal{H}(V)$ with its kernel, which is a function of $t$ and $\tau$, then $\mathcal{H}$ is defined by

$$
\mathcal{H}(V)=\int G(\boldsymbol{y}, t-\tau) V(\boldsymbol{y}) \mathrm{d} \boldsymbol{y}
$$

We will refer to $\mathcal{H}$ as the channel mapping.

At an abstract level, we will perform imaging by inverting the mapping $\mathcal{H}$

$$
\tilde{V}=\mathcal{H}^{-1} \mathcal{H}(V)
$$

By employing a set of transmit vectors $\left\{\boldsymbol{s}^{k}(t)\right\}$, and recording the corresponding measurement vectors $\left\{\boldsymbol{m}^{k}(t)\right\}$, we will obtain the action of $\mathcal{H}(V)$ on the space spanned by these transmit vectors. Once the channel $\mathcal{H}(V)$ is known, we will invert $\mathcal{H}$ using the Green's function for the background medium. This can all be performed in a Hilbert-space framework under mild conditions on the transmit waveforms and the reflectivity functions. We obtain a unique minimum-norm solution for the inversion problem by way of a pseudo inverse. ${ }^{13}$

\section{IMAGING UNDER DETERMINISTIC CONDITIONS}

First we will state our imaging algorithm for the case where there is no measurement noise.

Let the space of transmit vectors, $\mathrm{S}(0, T)^{m}$, be spanned by $\left\{\boldsymbol{s}^{k}(t)\right\}$. We assume that the waveforms are smooth* and supported on the finite interval $(0, T)$. Furthermore, we will assume that the reflectivity function $V$ is square integrable and non-zero only inside a sphere of finite radius. If we denote the measurement resulting from transmitting waveform $\boldsymbol{s}^{k}$ by $\boldsymbol{m}^{k}(t)$, we find that the adjoint of $\mathcal{H}$, denoted by $\mathcal{H}^{*}$, applied to the channel $\mathcal{H}(V)$ may be computed as $^{14}$

$$
\left[\mathcal{H}^{*} \mathcal{H}(V)\right](\boldsymbol{x})=\sum_{k}\left\langle\boldsymbol{m}^{k}, \mathcal{G}_{\boldsymbol{x}} \boldsymbol{s}^{k}\right\rangle,
$$

where the integral operator $\mathcal{G}_{\boldsymbol{x}}$ is defined as

$$
\mathcal{G}_{\boldsymbol{y}} \boldsymbol{s}(t)=\int G(\boldsymbol{y}, t-\tau) \boldsymbol{s}(\tau) \mathrm{d} \tau
$$

This is equivalent to transmitting orthogonal waveforms and matching (taking the inner-product between) the measurement and templates

$$
\mathcal{G}_{x} s^{k}(t):=\int G(\boldsymbol{x}, t-\tau) s^{k}(\tau) \mathrm{d} \tau
$$

and finally coherently adding up the results.

If we consider only finitely many transmit vectors, then we can show that $\mathcal{H}^{*} \mathcal{H}$ is compact. ${ }^{\dagger}$ Therefore, $\mathcal{H}^{*} \mathcal{H}$ is characterized by its action on a discrete set of orthogonal eigenfunctions.

Let $\left\{\left(\lambda_{p}, U_{p}\right)\right\}$ be the set of non-zero eigenvalues and associated eigenfunctions for $\mathcal{H}^{*} \mathcal{H}$

$$
\lambda_{p} U_{p}=\mathcal{H}^{*} \mathcal{H}\left(U_{p}\right)=\int \sum_{k}\left\langle\mathcal{G}_{x}^{*} \mathcal{G}_{\boldsymbol{y}} \boldsymbol{s}^{k}, \boldsymbol{s}^{k}\right\rangle U_{p}(\boldsymbol{y}) \mathrm{d} \boldsymbol{y}
$$

\footnotetext{
${ }^{*}$ Two times continuously differentiable is sufficient.

${ }^{\dagger}$ Note that even if $\mathcal{H}(V)$ in this case has finite-dimensional range, $\mathcal{H}$ does not; the space of HS operators between transmit vectors and measurements is infinite-dimensional. ${ }^{14}$
} 
We define a set of operators $\mathcal{U}_{p}$ which are related to $\mathcal{H}$ in the following manner:

$$
\mathcal{U}_{p}=\frac{1}{\sqrt{\lambda_{p}}} \mathcal{H}\left(U_{p}\right)
$$

From this we have obtained

$$
\mathcal{H}(V)=\sum_{p}\left\langle V, U_{p}\right\rangle \sqrt{\lambda_{p}} \mathcal{U}_{p}
$$

which is a singular-value decomposition (SVD) of the operator $\mathcal{H} .{ }^{13}$ By combining Eqns. (11) and (16), we arrive at our reconstruction formula

$$
\begin{aligned}
\tilde{V}(\boldsymbol{x}) & =\left(\mathcal{H}^{*} \mathcal{H}\right)^{-1} \mathcal{H}^{*} \mathcal{H}(V)(\boldsymbol{x}) \\
& =\sum_{p} \frac{1}{\sqrt{\lambda_{p}}} \sum_{k=1}^{K}\left\langle\boldsymbol{m}^{k}, \mathcal{U}_{p} \boldsymbol{s}^{k}\right\rangle U_{p}(\boldsymbol{x})
\end{aligned}
$$

where $\lambda_{p}, U_{p}$ and $\mathcal{U}_{p}$ are given by Eqns. (14) and (15). ${ }^{14}$

An algorithmic description of our reconstruction method is given below:

1. Select a set of orthonormal transmit vectors $\left\{\boldsymbol{s}_{k}\right\}$.

2. From the Green's function for the background medium we compute

$$
K(\boldsymbol{x}, \boldsymbol{y}):=\sum_{k}\left\langle\mathcal{G}_{\boldsymbol{x}}^{*} \mathcal{G}_{\boldsymbol{y}} \boldsymbol{s}_{k}, \boldsymbol{s}_{k}\right\rangle
$$

3. Determine the eigenvalues and eigenfunctions $\left(\lambda_{p}, U_{p}\right)$ of the integral operator with kernel $K(\boldsymbol{x}, \boldsymbol{y})$.

4. Determine the associated operators $\mathcal{U}_{p}$ of $\mathcal{H}$ according to Eq. (15).

5. Employ transmit vectors $\boldsymbol{s}^{k}$ and obtain the associated measurement vectors $\boldsymbol{m}^{k}$.

6. Match each measurement $\boldsymbol{m}^{k}$ with $\mathcal{U}_{p} \boldsymbol{s}^{k}$, and reconstruct according to Eq. (17).

\section{IMAGING UNDER UNCERTAINTY}

In this section we will consider measurements $\boldsymbol{m}^{k}(t)$ which are contaminated by additive noise $\boldsymbol{n}(t)$.

$$
\boldsymbol{m}^{k}(t)=\mathcal{H}(V) \boldsymbol{s}^{k}(t)+\boldsymbol{n}(t)
$$

We use the second-order statistical information about the reflectivity function in order to design a reconstruction method which is optimal for noise-contaminated measurements. Optimality is here defined in terms of mean square error (MSE). In order for our analysis to hold, we make a couple of assumptions about the statistics of the noise and the reflectivity function:

Assumption 1. The additive noise $\boldsymbol{n}(t)$ is assumed to be a zero-mean stochastic vector process with known covariance function:

1. The cross-covariance between the noise in the measurements at elements $i$ and $j$ is

$$
\mathrm{E}\left[n_{i}\left(t_{1}\right) \overline{n_{j}\left(t_{2}\right)}\right]=R_{i}\left(t_{1}, t_{2}\right) \delta_{i j}
$$


2. The covariance function satisfies one of the following conditions:

a) non-stationary noise: $\int R_{i}(t, t) \mathrm{d} t<\infty$

b) stationary noise: $R_{i}\left(t_{1}, t_{2}\right)=R_{i}\left(t_{2}-t_{1}, 0\right)$, and $\int\left|R_{i}(t, 0)\right| \mathrm{d} t<\infty$.

Assumption 2. The reflectivity function is a realization of a zero-mean random field with continuous covariance function

$$
R_{V}\left(\boldsymbol{y}_{1}, \boldsymbol{y}_{2}\right)=\mathrm{E}\left[V\left(\boldsymbol{y}_{1}\right) \overline{V\left(\boldsymbol{y}_{2}\right)}\right]
$$

Under Assumption 2, we can express the reflectivity function in terms of the Karhunen-Lóve (KL) expansion ${ }^{15}$

$$
V(\boldsymbol{x})=\sum_{r} c_{r} V_{r}(\boldsymbol{x}) .
$$

Here $\left\{V_{q}\right\}$ is a set of orthogonal functions, and $c_{q}$ are independent random variables.

Since $\mathcal{H}^{*}$ is invertible on the range of $\mathcal{H}$, we can without loss of generality assume that our linear reconstruction method is of the form:

$$
V^{\mathcal{B}}=\mathcal{B H}^{*}[\mathcal{H}(V)]
$$

where $\mathcal{B}$ is a suitable linear operator.

We define the MSE of the reconstruction as

$$
\operatorname{MSE}(\mathcal{B}):=\int \mathrm{E}\left[\left|V^{\mathcal{B}}(\boldsymbol{x})-P V(\boldsymbol{x})\right|^{2}\right] \mathrm{d} \boldsymbol{x},
$$

where $P$ is the projection onto the range of $\mathcal{H}^{*}$ :

$$
P V(\boldsymbol{x}):=\sum_{p}\left\langle V, U_{p}\right\rangle U_{p}(\boldsymbol{x})
$$

We obtain our imaging method for the noisy case from the $\mathcal{B}$ which minimizes the MSE as defined in Eq. (25). The resulting reconstruction is ${ }^{14}$

$$
\tilde{V}=\mathcal{M S}^{\frac{1}{2}}\left(\mathcal{S}^{\frac{1}{2}} \mathcal{M S}^{\frac{1}{2}}+\mathcal{E}\right)^{-1} \mathcal{S}^{-\frac{1}{2}} \mathcal{H}^{*}[\mathcal{H}(V)]
$$

where $\mathcal{H}^{*}$ applied to $\mathcal{H}(V)$ is computed by as in Eq. (11), but this time from noise-contaminated measurement vectors, and the operators $\mathcal{M}, \mathcal{S}$ and $\mathcal{E}$ have coefficients

$$
\begin{aligned}
(\mathcal{M})_{i j} & =\sum_{r} \mathrm{E}\left[\left|c_{r}\right|^{2}\right]\left\langle V_{r}, U_{i}\right\rangle \overline{\left\langle V_{r}, U_{j}\right\rangle} \\
(\mathcal{S})_{i j} & =\delta_{i j} \lambda_{i} \\
(\mathcal{E})_{i j} & =\sum_{k}\left\langle\mathcal{R}_{n} \mathcal{U}_{j} s^{k}, \mathcal{U}_{i} s^{k}\right\rangle .
\end{aligned}
$$

Here $\mathcal{R}_{\boldsymbol{n}}$ is a convolution operator where the kernel is a diagonal matrix with functions $R_{i}\left(t_{1}, t_{2}\right)$ from Eq. $(21)$ along the diagonal. We observe that Eq. (27) reduces to the result of Eq. (17) in the case when no additive noise is present. 


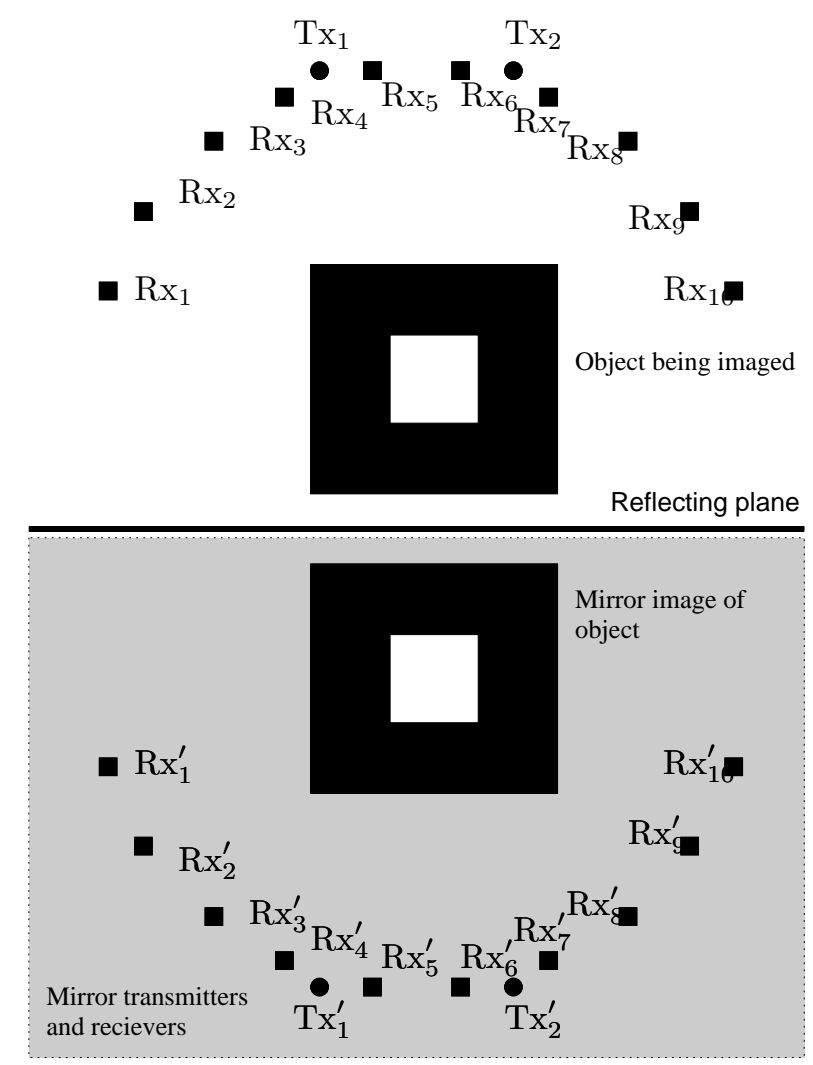

Figure 2. Distributed array with two transmitting elements (circles labelled $\mathrm{Tx}_{1}$ and $\mathrm{Tx}_{2}$ ), and ten receiving elements (small squares labelled from $\mathrm{Rx}_{1}$ to $\mathrm{Rx}_{10}$ ). The elements are placed at equidistant points along an arc with radius $10 \lambda$, where $\lambda$ is the wavelength corresponding to the center frequency of the transmitters. The target is indicated as a square with sides of $1.5 \lambda$, while the region of interest is $5 \lambda \times 5 \lambda$ around the target. The solid straight line indicates the location of a reflective mirror in our simple multi-path scenario. A grey box frames the mirror image of the object, as well as the mirror transmitters (labelled $\mathrm{Tx}_{1}^{\prime}$ and $\mathrm{Tx}_{2}^{\prime}$ ) and mirror receivers (labelled from $\mathrm{Rx}_{1}^{\prime}$ to $\mathrm{Rx}_{10}^{\prime}$ ).

\section{NUMERICAL SIMULATIONS}

In order to demonstrate the performance of our reconstruction method, we conducted a set of numerical simulations for two different scenarios. First we simulated scattering from an object in a free-space background. Then we performed another simulation where we inserted a mirror surface behind the object to be imaged (see Fig. 2).

We used a distributed aperture with two transmit elements and 10 receive elements. The antenna elements were equally spaced on a semi-circle with radius $10 \lambda$. The object which we wanted to image was a square with sides of $1.5 \lambda$. We reconstructed this reflectivity in a region of $5 \lambda \times 5 \lambda$ around the square. Figure 2 gives an illustration of our simulated scenario.

In all experiments the two transmitters operated simultaneously: transmitter 1 emitted a linear up-chirp $\sin \left(\omega_{0}\left[t+\alpha t^{2}\right]\right)$, while transmitter 2 simultaneously emitted a linear down-chirp $\sin \left(\omega_{0}\left[t-\alpha t^{2}\right]\right)$. Thus, there was an inherent ambiguity in the reflected waveforms as to the source of the energy.

We performed numerical experiments to demonstrate the performance of our image reconstruction method for the following cases: 1) free space, 2) multi-path environment, 3) measurements with colored noise.

\subsection{Noise-free simulations}

For the experiments 1) and 2) the measurements were free of noise, and the reflectivity function was deterministic. We therefore employed the reconstruction formula from Eq. (17). In this case we reconstruct a projection of 

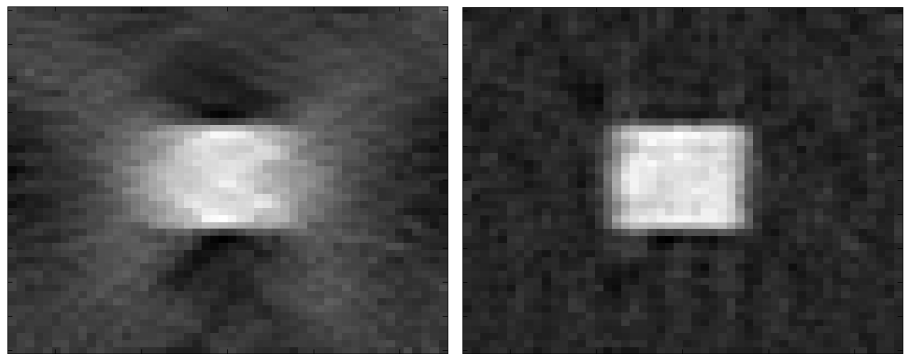

Figure 3. Image reconstructed using our method from Eq. (17). In this case we employed 2 transmitters and 10 receivers. For the free-space case (left) the reconstruction error was $-5.7 \mathrm{~dB}$. In the multipath case (right) we get a reconstruction error of $-11.2 \mathrm{~dB}$.

the reflectivity function onto the range of $\mathcal{H}^{*}$. It is of interest to see how well this approximates the true reflectivity function when we have a distributed aperture. We use the following relative $\mathrm{L}^{2}$ norm to quantify the reconstruction error

$$
\text { Error }:=10 \log \left(\frac{\int\left|\left[\mathcal{H}^{-1} \mathcal{H}(V)\right](\boldsymbol{x})-V(\boldsymbol{x})\right|^{2} \mathrm{~d} \boldsymbol{x}}{\int|V(\boldsymbol{x})|^{2} \mathrm{~d} \boldsymbol{x}}\right)
$$

1) Free Space In the left panel of Fig. 3, we see a reconstruction for the free-space scenario using our reconstruction method when we have 2 transmitters and 10 receivers distributed as indicated in Fig. 2. The reconstruction error as defined in Eq. (31) is here $-5.7 \mathrm{~dB}$.

2) Multipath The right panel of Fig. 3 shows reconstruction for our multipath scenario when we have 2 transmitters and 10 receivers distributed as indicated in Fig. 2. The reconstruction error is in this case $-11.2 \mathrm{~dB}$.

\subsection{Simulations with noise}

The results for simulation case 3) were obtained using the reconstruction formula in Eq. (27). The overall simulation setup is the same as for the deterministic case. However, here we add noise to our scattering measurements, and employ a stochastic model for the reflectivity function.

In our experiment we considered a wide-band $1 / \omega$-type noise model. ${ }^{16}$ The noise was generated by filtering white noise in the frequency domain to obtain a power spectrum proportional to

$$
\frac{1}{1+\omega^{2}} \text {. }
$$

Furthermore, we considered a square object with unknown scattering strength $c$, i.e.,

$$
V(\boldsymbol{x})=c \Xi(1.5 \lambda \boldsymbol{x}) .
$$

Here $\Xi$ is 1 on the unit square, and the scattering strength $c$ is normally distributed with unit variance.

We define the signal-to-noise ratio (SNR) as follows

$$
\mathrm{SNR}:=\sup _{t} \sqrt{\frac{\mathrm{E}\left[\left|\mathcal{H}(V) \boldsymbol{s}^{k}(t)\right|^{2}\right]}{\mathrm{E}\left[|\boldsymbol{n}(t)|^{2}\right]}},
$$

where $\boldsymbol{n}(t)$ is the additive noise process. To determine this we estimated $\mathrm{E}\left[\left|\mathcal{H}(V) \boldsymbol{s}^{k}(t)\right|^{2}\right]$ by averaging over 50 realizations of the reflectivity function, and found its peak value. We then scaled the additive noise variance to obtain the desired SNR.

In order to perform the inversion we needed to determine the operators $\mathcal{M}, \mathcal{S}$ and $\mathcal{E}$ as defined in Eqns. (28)(30) For this we used the fact that we already had computed the eigenfunctions $U_{p}$ of $\mathcal{H}^{*} \mathcal{H}$ in order to perform our noise-free reconstructions. 
- The coefficients for the operator $\mathcal{M}$, as defined in Eq. (28), were obtained by numerically projecting our model for the reflectivity function onto the already-computed eigenfunctions $U_{p}$ of $\mathcal{H}^{*} \mathcal{H}$. In particular we projected our square object in Eq. (33) onto these eigenfunctions, and used $\mathrm{E}\left[|c|^{2}\right]=1$.

- The elements of the diagonal operator $\mathcal{S}$ were obtained from previous computations of the eigenvalues associated with the eigenfunctions of $\mathcal{H}^{*} \mathcal{H}$.

- To obtain $\mathcal{E}$, we first computed $\mathcal{U}_{j} s_{k}(t)$ by performing noise-free scattering simulations from the reflectivity function $U_{j}$. Furthermore, we computed $\mathcal{R}_{n} \mathcal{U}_{j} s_{k}(t)$ by applying to each vector element of this simulated scattering a filter with the impulse response $\frac{1}{1+\omega^{2}}$ from Eq. (32). Finally, the element $(\mathcal{E})_{i j}$ was obtained by taking the inner product between this filtered scattering simulation and an un-filtered scattering simulation from the reflectivity $U_{i}$, and summing over all our transmit vectors. For a particular SNR, we multiplied the whole operator by a constant which corresponded to the scaling applied to the additive noise.

We characterize the reconstruction quality in the presence of additive noise using a relative mean-square error expressed in a decibel scale:

$$
\operatorname{MSE}(\mathcal{W})=10 \log \left(\frac{\int \mathrm{E}\left[\left|\left[\mathcal{H}^{-1} \mathcal{H}(V)\right](\boldsymbol{x})-P V(\boldsymbol{x})\right|^{2}\right] \mathrm{d} \boldsymbol{x}}{\int \mathrm{E}\left[|V(\boldsymbol{x})|^{2}\right] \mathrm{d} \boldsymbol{x}}\right) .
$$

This is clearly equivalent to using the MSE as defined in Eq. (25).

When computing the MSE according to Eq. (35), we need the projection $P V$ of the reflectivity function onto the range of $\mathcal{H}^{*}$. We obtained this from the reconstructions in experiments 1) and 2), i.e., reconstructions without noise. The value for $\int \mathrm{E}\left[\left|\left[\mathcal{H}^{-1} \mathcal{H}(V)\right](\boldsymbol{x})-P V(\boldsymbol{x})\right|^{2}\right] \mathrm{d} \boldsymbol{x}$ was then estimated from 10 different reconstructions using different noise realizations. Finally, we see that we can compute $\int \mathrm{E}\left[|V(\boldsymbol{x})|^{2}\right]$ from Eq. (33) directly. This gives us our estimated reconstruction error for the statistical reconstruction.

3) Simulated scattering with noise Figure 4 shows examples of reconstruction in the presence of additive noise. The reconstruction is based on scattering from a unit square with unit scattering strength. The noise was added so that the measurements had an SNR of 20. In the top left panel of Figure 4 we see a reconstruction for our free-space scenario, while in the top right panel we have a reconstruction for our multi-path scenario. In order to show the necessity of taking the additive noise into account, we have also included a deterministic free-space reconstruction in the lower left panel of Figure 4. In the lower right panel of Figure 4 we see the MSE as a function of SNR. Here, the solid line and dash-dot line denote statistical reconstruction in the free-space case and the multi-path case, respectively. The MSE of the deterministic free-space reconstruction is shown as dotted line. The MSE was estimated according to Eq. (35) by averaging over 10 realizations of the scattering potential and the additive noise for each SNR. The MSE was then normalized relative to the free-space statistical reconstruction.

\section{DISCUSSION AND CONCLUDING REMARKS}

We see that multipath improves the reconstruction when we apply our reconstruction method. In particular we note that the vertical edges are much sharper when multipath is present. By using the method of images, we can show that our multipath scenario may be viewed as a free-space scenario with additional transmitters and receivers. ${ }^{14}$ Thus if we can exploit the multipath returns properly, these additional antenna elements provide illumination of the object from new directions. If we employ a ray approximation to our propagating field, then this phenomenon is explained by the fact that only edges perpendicular to the bisector between the incident ray and the reflected ray are visible. ${ }^{17}$ A similar effect was also observed previous work. ${ }^{11}$

The MSE that we have plotted in Figure 4 is measured relative to the noise-free reconstruction in each case. Thus, the MSE of the multi-path reconstruction is judged against a much better reference than the freespace reconstruction ( see Figure 3). As a result, the MSE of the multi-path does not correctly portray the reconstruction improvement over the free-space reconstruction relative to the true object. 

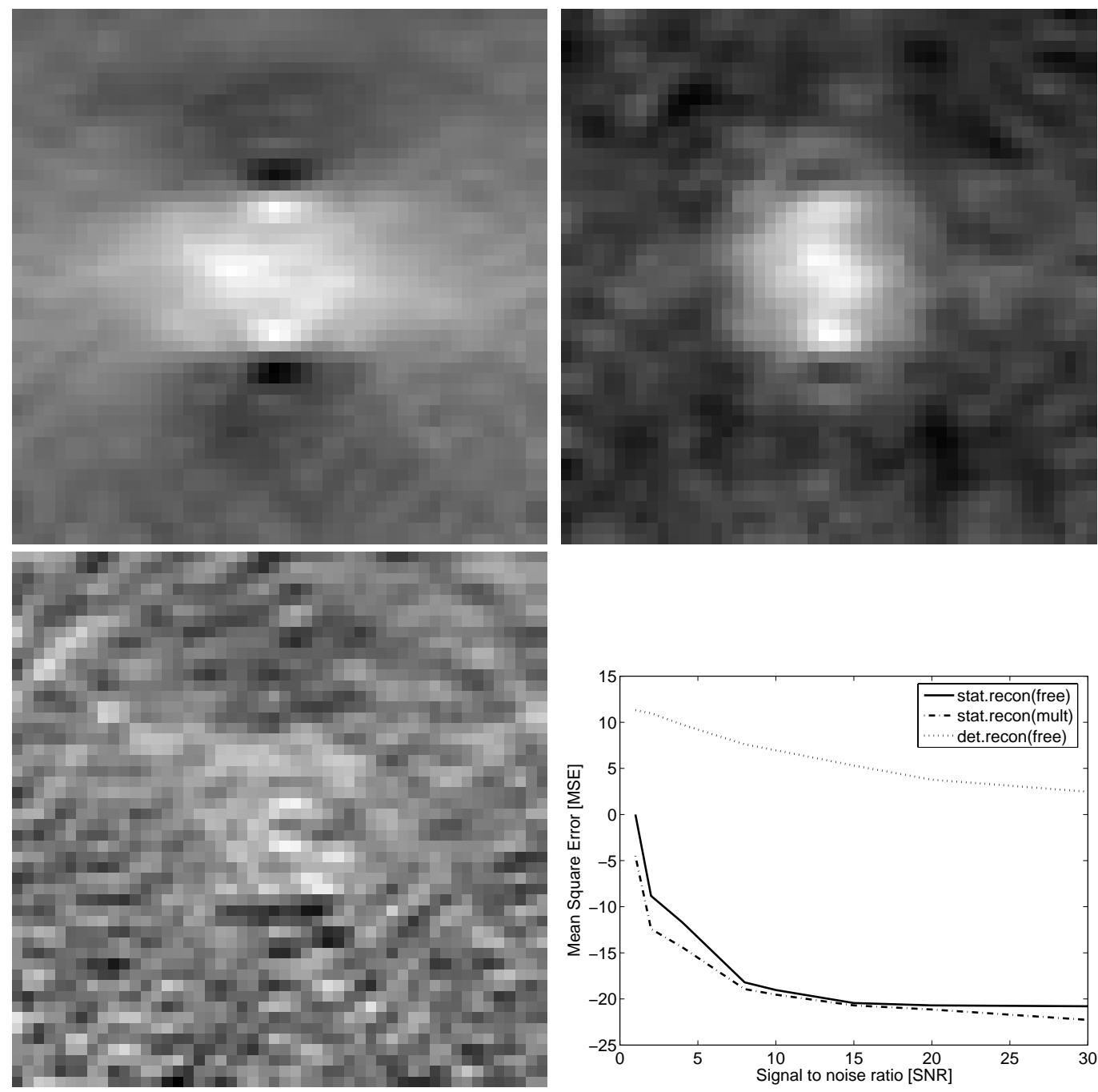

Figure 4. Reconstructed images in the presence of additive noise. Top left: statistical reconstruction in free space with $\mathrm{SNR}=20$. Top right: statistical reconstruction in multi-path with $\mathrm{SNR}=20$. Bottom left: deterministic reconstruction in free space with $\mathrm{SNR}=20$. Bottom right: $\mathrm{MSE}$ in the reconstructed images as a function of SNR.

The functions $\left\{U_{p}\right\}$ provide an orthogonal basis for the reconstruction. Furthermore, by virtue of being eigenfunctions of $\mathcal{H}^{*} \mathcal{H}$, they yield a representation of the reflectivity function where the terms associated with large eigenvalues correspond to the most visible parts. In this sense, computing a reconstruction based on only the terms corresponding to the largest eigenfunctions will yield a compressed representation of the image which contain only the most significant features - significance being quantified by their $\mathrm{L}^{2}$ norm. Additionally, by orthogonality each additional term which is computed for our imaging algorithm will provide reconstruction of the reflectivity function in a new subspace of $\mathrm{L}^{2}(\Omega)$. In this sense our reconstruction is efficient.

If the template fields $\mathcal{U}_{p} s^{k}$ are computed off-line, the inversion is efficiently implemented as a set of innerproducts independently computed for each array element. This means that a significant part of the computation can be performed in a decentralized fashion on each array element. Since the reconstructed reflectivity function is expanded in terms of the basis functions, only the coefficients in this expansion need to be communicated from each element. This combination of distributed processing and compressed information representation is desirable in applications where the communication bandwidth from each aperture element is limited.

If the additive noise is white, then $\mathcal{E}$ is a diagonal operator. Furthermore, if our distributed aperture is 
organized such that the basis functions $\left\{U_{p}\right\}$ that are used for reconstruction corresponds to the basis functions $\left\{V_{p}\right\}$ for our reflectivity function model, then we see that the optimal reconstruction in the presence of noise is obtained by Tikhonov regularization of the inversion formula given in Eq. (17).

Note that if the reflectivity function has non-zero mean, the MMSE reconstruction formula of Eq. (27) can be obtained by using a minimum variance estimate criterion. In this case the MSE includes a bias term in addition to the one given in Eq. $(25){ }^{16}$

\section{Acknowledgments}

We are grateful to Air Force Office of Scientific Research ${ }^{\ddagger}$ (AFOSR) and the Defense Advanced Research Projects Agency (DARPA) for supporting this work under the agreements FA9550-04-1-0223, FA9550-07-1-0363, FA955006-1-0017 and FA8750-05-2-0285.

\section{REFERENCES}

[1] F. Natterer and F. Wbbeling, Mathematical Methods in Image Reconstruction, SIAM, USA, 2001.

[2] R. Adve, R. Schneibler, G. Genello, and P. Antonik, "Waveform-space-time adaptive processing for distributed aperture radars," in Proc. 2005 IEEE Radar Conf., pp. 93-97, 2005.

[3] R. Adve, R. Schneibler, and R. McMillan, "Adaptive space/frequency processing for distributed apertures," in Proc. 2003 IEEE Radar Conf., pp. 160-164, 2003.

[4] R. Adve, "Sub-optimal adaptive processing for distributed aperture radars," in Proc. 2nd Waveform Diversity Workshop, pp. 160-164, (Verona, NY), 2003.

[5] E. Fishler, A. Haimovich, R. Blum, L. Cimini, D. Chizhik, and R. Valenzuela, "Spatial diversity in radars - models and detection performance," IEEE Trans. Sign. Processing 54(3), pp. 823-238, 2006.

[6] I. Bekkerman and J. Tabrikian, "Target detection and localization using MIMO radars and sonars," IEEE Tran. Signal Proc. 54(10), pp. 3873-3883, 2006.

[7] A. Fletcher and F. Robey, "Performance bounds for adaprive coherence of sparse array radar," in Proc. of 11th. Conf. Adaptive Sensors Array Processing, Lexington (MA), March 2003.

[8] D. Rabideau, "Ubiquitous mimo digital array radar," in Proc. 37th Asilomar Conf. Signals, Systems, Computers, 2003.

[9] E. Bond, S. Hagness, and B. Veen, "Microwave imaging via space-time beamforming for early detection of breast cancer," IEEE Trans. Ant. Prop. 51(8), pp. 1690-1705, 2003.

[10] M. Dennison and A. Devaney, "Inverse scattering in inhomogeneous background media: II. multi-frequency case and svd formulation," Inverse Problems 20, pp. 1307-1324, 2004.

[11] A. Devaney and M. Dennison, "Inverse scattering in inhomogeneous background media," Inverse Problems 19, pp. 855-870, 2003.

[12] B. Yazıcı and G. Xie, "Wideband extended range-Doppler imaging and waveform design in the presence of clutter and noise," IEEE Trans. Inf. Theory 52(10), 2006.

[13] H. Engl, M. Hanke, and A. Neubauer, Regularization of inverse problems, Kluwer Academic Publishers, Netherlands, 2000.

[14] T. Varslot, B. Yazıcı, and M. Cheney, "Wide-band pulse-echo imaging with distributed apertures in multipath environments," Inverse Problems , 2008. (submitted).

[15] P. Todorovic, An introduction to stochastic processes and their applications, Statistics, Springer-Verlag, New York, 1992.

[16] B. Yazıcı, M. Cheney, and C. E. Yarman, "Synthetic aperture inversion for an arbitrary flight trajectory in the presence of noise and clutter," Inverse Problems 22(5), pp. 1705-1729, 2006.

[17] C. E. Yarman, B. Yazici, and M. Cheney, "Bistatic synthetic aperture radar imaging with arbitrary trajectories," IEEE Transactions in Image Processing 17, pp. 84-93, January 2008.

\footnotetext{
${ }^{\ddagger}$ Consequently, the US Government is authorized to reproduce and distribute reprints for governmental purposes notwithstanding any copyright notation thereon. The views and conclusions contained herein are those of the authors and should not be interpreted as necessarily representing the official policies or endorsements, either expressed or implied, of the Air Force Research Laboratory or the US Government.
} 